\title{
Comparative metabolomic profiling in the roots of salt-tolerant and salt-intolerant maize cultivars treated with $\mathrm{NaCl}$ stress
}

\author{
J.Y. YUE, L.H. WANG, X.T. DOU, Y.J. WANG, and H.Z. WANG* \\ Tianjin Key Laboratory of Animal and Plant Resistance, Tianjin Normal University, \\ Tianjin 300387, P.R. China
}

\begin{abstract}
Maize crops are sensitive to $\mathrm{NaCl}$ stress, which is one of the most harmful abiotic stresses affecting agricultural productivity. To gain further insights into the differential metabolic responses to $\mathrm{NaCl}$ stress, we employed metabolomics and physiological approaches to understand the response of salt-tolerant (PH6WC) and sensitive (PH4CV) cultivars of maize. Salt stress caused a significant reduction in root growth, lower root numbers, softened roots, leaf etiolation, inhibition of leaf formation, and decreased shoot height and stem width in both the tolerant and sensitive genotypes compared with the control. These morphological characteristics increased with the progression of the $\mathrm{NaCl}$ concentration, however, they were less prominent in the salt-tolerant genotype. Evans blue staining demonstrated that $\mathrm{NaCl}$-induced root cell death, and the root cells of 'PH4CV' were almost completely dead following $9 \mathrm{~d}$ of exposure to $100 \mathrm{mM} \mathrm{NaCl}$. Under treatment with $100 \mathrm{mM} \mathrm{NaCl}, 79$ compounds in the roots of 'PH4CV' were identified as being significant metabolites, and 85 compounds were identified as being significant metabolites in the roots of 'PH6WC'. The NaCl-induced changes in the metabolomes of these two maize cultivars indicate that 80 root-based compounds were different between $\mathrm{NaCl}$-treated plants and controls. Among these metabolites, 30 were found in both maize cultivars when responding to $\mathrm{NaCl}$ stress. These compounds were associated with the metabolism of some basic compounds such as cis-9-palmitoleic acid, L-pyroglutamic acid, galactinol, deoxyadenosine, and adenine. The changing abundance of the 30 metabolites was not completely consistent in 'PH4CV' and 'PH6WC'. Glucose metabolism was exclusively induced by $\mathrm{NaCl}$ in the 'PH4CV' maize seedlings whereas nucleic acid metabolism was more significant in the 'PH6WC' maize seedlings in response to $\mathrm{NaCl}$ stress. Overall, 'PH6WC' and 'PH4CV' responded differently to $\mathrm{NaCl}$ stress, and this information is helpful in understanding how maize seedlings respond to this type of abiotic stress.
\end{abstract}

Additional key words: cell death, glucose metabolism, leaf elongation, nucleic acid metabolism, root morphology, Zea mays.

\section{Introduction}

Salt stress is a major enviromental factor that severely endangers the growth and distribution of plants, especially in arid and semi-arid areas (Jung et al. 2017, El-Katony et al. 2019). Salt stress inhibits plant water uptake, and toxic concentrations of $\mathrm{Na}^{+}$and $\mathrm{Cl}^{-}$often accumulated in the cytosol (Jiang et al. 2019). Both the ion toxicity and osmotic stress induces subsequent oxidative stress (Flowers et al. 2015, Abd El-Gawad et al. 2016, Zhang et al. 2018, Carillo et al. 2019). Plant responses to salinity are not only dependent on plant species but also on the duration of salt exposure as well as the concentration and the type of salts (Carillo et al. 2019). At short-term salinity, plant growth inhibition can be explained by reduced leaf elongation through decrease in pressure potential in cell of expanding tissues while long-term salinity affects many different aspects of growth, including those associated with water stress and those specific to $\mathrm{NaCl}$ (Shabala et al. 2016). Thus, when salinity tolerance and physiological responses are evaluated in species that have evolved under naturally occurring salinity stress, both the intensity and the time course over which the stress is imposed should be considered. According to previous reports, global warming and unreasonable irrigation of arable soil can further worsen salination in soil and massively decrease in production of salt-sensitive crops (Ceccarini et al. 2019, Issaoui et al. 2019).

Maize is characterized by high yield and a wide geographical scope for growth (Gao et al. 2016). Its

Submitted 9 February 2020, last revision 22 May 2020, accepted 28 May 2020.

Acknowledgments: We thank the other members of our laboratory for help in the research and for insightful remarks. This work was supported by the National Science Foundation of China (No. 31501234 and 31971829), the Natural Science Foundation of Tianjin (18JCYBJC30300, 17JCZDJC33800), and the youth talent support program of Tianjin Normal University (043135202RC1702). Thank the referees for helpful comments.

* Corresponding author; fax: (+86) 22 2376 6539, e-mail: 421052455@qq.com 
kernels contain a diverse range of phenols and carotenoids, and one unique constituent (GNA-maize) which has the potential to inhibit the activity of the AIDS virus. However, a number of studies have indicated that salt-sensitive maize cultivars are harmed and produce lower yields when salt concentration in soil solution reach $150 \mathrm{mM}$ (Rouf Shah et al. 2016, Du et al. 2017, Luo et al. 2017b). In order to increase maize yields, many researchers have set out to study its salt tolerance mechanism from the perspectives of physiology, biochemistry, and molecular biology and thus developed novel salt-resistant germplasms. However, because of the complicated relationship between maize yield and soil salinity, the current level of information about the salt tolerance mechanism of maize is far from comprehensive (Juhos et al. 2015).

The emergence of "omics" has become a research hotspot in different scientific fields including metabolomics, proteomics, genomics, and transcriptomics (Singh et al. 2015, You et al. 2019). In recent years, some omicsoriented reports concerning the salt tolerance mechanism of maize have been produced, but these focus more on regulatory changes in the proteome and transcriptome of maize under salt stress and rarely report the metabolome of salt tolerance or sensitive maize (Richter et al. 2015, Cai et al. 2017, Du et al. 2017, Luo et al. 2017a, Wang et al. 2017, Trevisan et al. 2019). The mechanisms of salt tolerance in plants are not fully understood. Metabolomics is the scientific study of internal changes in substances present in biological organisms to adapt to disturbances or stimuli in the external environment. Featuring "systematicdynamic-integrated-analytical" principles, metabolomics accords with the dynamic process of plants in response to environmental change (You et al. 2019). Therefore, metabolomics-based approaches may be used to facilitate a systematic understanding of the salt stress-related mechanism in maize.

$\mathrm{NaCl}$ generally dissolves easily in soil. In nonsaline conditions, $\mathrm{NaCl}$ can be beneficial for plants. When present in a range of $0.2-2 \mathrm{mg} \mathrm{g}^{-1}$ (f.m.), it can stabilize the oxygen evolving complex of photosystem II, maintain cell membranes electric potential, and regulate tonoplast $\mathrm{H}^{+}$-ATPase, $\mathrm{pH}$-stat, amylases, and asparagine synthetase. However, when its concentration is more than $4 \mathrm{mg} \mathrm{g}^{-1}$ (f.m.), $\mathrm{NaCl}$ can be toxic impairing photosystem II quantum yield and photosynthetic electron transport rate and consequently causing other physiological dysfunctions in plants (Carillo et al. 2019 and references therein). Harmful changes in the external environment cause severe damage to plants in their early stages of development, and seedling growth determines the subsequent productivity of the crop. Therefore, investigation of the mechanism underlying seedling responses to $\mathrm{NaCl}$ stress is of critical significance (Farooq et al. 2015, Shelke et al. 2017, Zezulka et al. 2019). In the present study, the salt-sensitive (PH4CV) and salt tolerant (PH6WC) maize genotypes were used as experimental materials to compare the variation of phenotypic characteristics and root activity under $\mathrm{NaCl}$ stress. The metabolic profiling in two maize genotypes with contrasting ability to cope with $\mathrm{NaCl}$ stress was compared, the key metabolic pathways involved in
$\mathrm{NaCl}$ response in maize were identified by metabolomic analysis. The outcomes of this study can provide molecular evidence for elucidating the molecular mechanism of plant responses to salt stress.

\section{Materials and methods}

Plants, growth conditions, and treatments: The test species were salt-sensitive $\mathrm{PH} 4 \mathrm{CV}$ and salt-tolerant PH6WC maize (Zea mays L.) genotypes. 'PH6WC' and 'PH4CV' were the female and male parents of maize hybrid Xianyu335 bred by Tieling Pioneer Seed Research Co. Ltd. 'PH6WC' was bred from the crossing PH01N $\times$ $\mathrm{PH} 09 \mathrm{~B}$ from Reid population. 'PH4CV' was bred from the crossing $\mathrm{PH} 7 \mathrm{~V} 0 \times \mathrm{PHBE} 2$ from Lancaster population. These two maize PH4CV and PH6WC cultivars were preliminarily selected from 23 cultivars in the seedling stage where 'PH4CV' was found as the salt-sensitive cultivar and 'PH6WC' as the salt-tolerant cultivar. Seeds were firstly washed with tap water then rinsed with distilled water thrice before being soaked in distilled water for $12 \mathrm{~h}$. The seeds were then transferred to a culture basin covered with a wet gauze. A small amount of distilled water was sprayed onto the seeds for culture at ambient temperature. The distilled water was changed every $24 \mathrm{~h}$ until the maize grew to the one-leaf-and-one-bud stage. The uniform seedlings were transplanted into a 1/10 Hoagland solution for further culture. The nutrient solution was changed every $2 \mathrm{~d}$ until the maize grew to the three-leaf-and-onebud stage.

Each genotype was planted in plastic pots $(50 \mathrm{~cm}$ in diameter and $40 \mathrm{~cm}$ in height; 10 plants per pot) containing the $1 / 10$ Hoagland nutrient solution $(\mathrm{pH} 5.2)$ with $0 \mathrm{mM} \mathrm{NaCl}$ to form the control group and various concentrations of $\mathrm{NaCl}(10,25,50$, and $100 \mathrm{mM})$ to form the salt stress treatment group. During the experiment, salinity gradually increased to avoid salt shock to the plants. $\mathrm{NaCl}$ concentrations increased in $25 \mathrm{mM}$ increments every second day for salt adaption until the final concentration was reached. The nutrient solution was exchanged at $1 \mathrm{~d}$ intervals.

Determination of root activity: Evans blue staining can be used to perform fast identification of dead cells. Deeper staining of more sites indicates less cell activity in the root. Evans blue staining liquid can penetrate into the cells through damaged membrane and combine with the proteins to stain them blue (Gholami et al. 2018). The staining approach was modified based on the methods proposed by Baker (1994) and Chalivendra (2017).

Three seedlings were harvested from both non- $\mathrm{NaCl}$ -treated and $\mathrm{NaCl}$-treated groups at $0,1,3,6$, and $9 \mathrm{~d}$ after the onset of $\mathrm{NaCl}$ stress. They were washed with tap water, distilled water, and deionized water, respectively, and then dried with filter paper. The root tips $(1.5 \mathrm{~cm})$ were sampled and placed in $0.25 \%$ Evans blue staining liquid for $8 \mathrm{~min}$ in darkness. Then, the tissues were rinsed with deionized water until it became colorless. After $1 \mathrm{~h}$, the tissues were photographed under a stereoscopic microscope (Nikon 
C-fled2, Nikon, Tokyo, Japan) to record the staining of the roots.

The stained portions of the seeding roots were removed and cut into pieces before being added to Evans blue extracting liquid (at the ratio of $1 \mathrm{mg}$ root tissue to $0.01 \mathrm{~cm}^{3}$ of extracting liquid prepared with $50 \%(\mathrm{v} / \mathrm{v})$ methyl alcohol, $1 \%(\mathrm{~m} / \mathrm{v})$ sodium dodecyl sulfate (SDS), and water. Extraction was performed in a water bath at $60{ }^{\circ} \mathrm{C}$ for $1-2 \mathrm{~h}$ until the tissues became completely colorless. Then, the extracting liquid was washed with flowing water at ambient temperature. A microplate reader was used to measure the absorbance at $600 \mathrm{~nm}$.

Metabolomic assay - sample collection and preparation: Ammonium acetate, ammonium hydroxide, ammonium fluoride, and formic acid were purchased from Sigma Aldrich (Saint Louis, MO, USA). Acetonitrile was purchased from Merck (Shanghai, China).

Seven seedlings of salt-sensitive and salt-tolerant maize cultivars were randomly selected from three pots where they were grown under control conditions and $100 \mathrm{mM} \mathrm{NaCl}$ treatment for $6 \mathrm{~d}$. After being washed with tap water, distilled water, and deionized water in turn, the seedlings were gently dried with filter paper and cut quickly into $2-\mathrm{cm}$ pieces of root tips. From each sample, $0.6 \mathrm{~g}$ of tissue was weighed and placed into $15-\mathrm{m}^{3}$ Eppendorf tubes, frozen in liquid nitrogen and then kept in a freezer at $-80{ }^{\circ} \mathrm{C}$. Samples from each group were six times. Moreover, control samples were prepared for the purpose of stabilizing and calibrating experimental instruments and approaches. The samples from the saltsensitive 'PH4CV' control group were labeled as $\mathrm{C} 1$ and those from the treatment group as T1, samples from the salt-tolerant 'PH6WC' control group were labeled as C2 and those from the treatment group as T2.

The samples frozen at $-80{ }^{\circ} \mathrm{C}$ were removed and ground under liquid nitrogen. Further, $60 \mathrm{mg}$ was weighed out and added to $1 \mathrm{~cm}^{3}$ of methanol:acetonitrile:water solution $(2: 2: 1, \mathrm{v} / \mathrm{v})$ before being shaken for $60 \mathrm{~s}$ on a vortex shaker and underwent to a $30 \mathrm{~min}$ low-temperature ultrasound. Afterwards, the samples were left at $-20^{\circ} \mathrm{C}$ to settle the proteins, filtered with a filter pipe, and centrifuged at $14000 \mathrm{~g}$ and $4{ }^{\circ} \mathrm{C}$ for $20 \mathrm{~min}$. Finally, the supernatant was removed for freezing and drying, and the samples were kept at $-80^{\circ} \mathrm{C}$.

Analysis by liquid chromatography - mass spectrometry / mass spectrometry analysis: Analyses were performed using an ultra high performance liquid chromatography system (1290 Infinity LC, Agilent Technologies, Santa Clara, CA, USA) coupled to a quadrupole time-of-flight system (AB Sciex TripleTOF 6600, Shanghai Applied Protein Technology Co., Shanghai, China).

For hydrophilic interaction liquid chromatography separation, samples were analyzed using a $2.1 \mathrm{~mm}$ $\times 100 \mathrm{~mm}$ ACQUIY UPLC BEH $1.7 \mu \mathrm{m}$ column (Waters, Wexford, Ireland). In both electron spray ionization positive and negative modes, the mobile phase contained $\mathrm{A}=25 \mathrm{mM}$ ammonium acetate and $25 \mathrm{mM}$ ammonium hydroxide in water and $\mathrm{B}=$ acetonitrile. The gradient was $95 \% \mathrm{~B}$ for $1 \mathrm{~min}$, linearly reduced to $65 \%$ over $13 \mathrm{~min}$, and then reduced to $40 \%$ over $2 \mathrm{~min}$, and maintained for $2 \mathrm{~min}$, then increased to $95 \%$ over $0.1 \mathrm{~min}$, followed by a 4.9 min re-equilibration period.

The electrospray ionization source conditions were set as follows: ion source gas1 (Gas1) as 60, ion source gas2 (Gas2) as 60, curtain gas (CUR) as 30, source temperature $600{ }^{\circ} \mathrm{C}$, ion spray voltage floating $\pm 5500 \mathrm{~V}$. In mass spectrometry only acquisition, the instrument was set to acquire data over the $\mathrm{m} / \mathrm{z}$ range $60-1000 \mathrm{Da}$ and the accumulation time for a time-of-flight mass spectrometry scan was set at $0.20 \mathrm{~s} /$ spectra. In auto mass spectrometry/ mass spectrometry acquisition, the instrument was set to acquire data over the $\mathrm{m} / \mathrm{z}$ range $2-1000 \mathrm{Da}$ and the accumulation time for the product ion scan was set at $0.05 \mathrm{~s} / \mathrm{spectra}$. The product ion scan was acquired using information-dependent acquisition with the high sensitivity mode selected. The parameters were set as follows: the collision energy was fixed at $35 \pm 15 \mathrm{eV}$, declustering potential $60 \mathrm{~V}(+)$ and $-60 \mathrm{~V}(-)$, exclude isotopes within $4 \mathrm{Da}$, candidate ions to monitor per cycle: 6 .

Data analysis: For root activity experiments, three replicates were performed. All data measured were subjected to analysis of variance. The mean differences were compared using the Duncan's multiple range test. Differences with $P \leq 0.05$ were considered significant.

For metabolic data analysis, the raw mass spectrometry data (wiff.scan files) were converted to MzXML files using ProteoWizard MSConvert before importing into a freely available XCMS software. For peak picking, the following parameters were used: centWave $\mathrm{m} / \mathrm{z}=25 \mathrm{ppm}$, peakwidth $=\mathrm{c}(10,60)$, and prefilter $=\mathrm{c}(10,100)$. For peak grouping, $\mathrm{bw}=5, \mathrm{mzwid}=0.025$, and $\operatorname{minfrac}=0.5$ were used. CAMERA (collection of algorithms of metabolite profile annotation) was used to annotate isotopes and adducts. In the extracted ion features, only the variables with more than $50 \%$ of the nonzero measurement values in at least one group were kept. Identification of metabolites was performed by comparing $\mathrm{m} / \mathrm{z}$ values $(<25 \mathrm{ppm})$ and mass spectrometry / mass spectrometry spectra with an in-house database established with available authentic standards.

After normalizing to total peak intensity, the processed data were uploaded before being imported into SIMCA-P (v. 14.1, Umetrics, Umea, Sweden), where they were subjected to multivariate data analysis, including Paretoscaled principal component analysis and orthogonal partial least squares discriminant analysis (OPLS-DA). Sevenfold cross-validation and response permutation tests were used to evaluate the robustness of the model. The variable importance in the projection (VIP) value of each variable in the OPLS-DA model was calculated to indicate its contribution to the classification. Metabolites with the VIP value $>1$ were further applied to the Student's $t$-test at the univariate level to measure the significance of each metabolite. Differences with $P \leq 0.05$ were considered as significant. 


\section{Results}

During the experimental period, $\mathrm{NaCl}$ stress caused a significant reduction in the root growth, lowered root numbers, softened roots, caused leaf etiolation, inhibited leaf formation, and decreased shoot height and stem width in both the tolerant and sensitive genotypes compared with the control and the effects increased with the progression of $\mathrm{NaCl}$ stress (Fig. 1 Suppl.). The sensitive genotype showed significant changes in these morphological characteristics in response to $\mathrm{NaCl}$ stress compared with plants grown under control conditions. However, the morphological changes were less apparent in the tolerant genotype. The 'PH4CV' maize seedlings withered following $9 \mathrm{~d}$ of $\mathrm{NaCl}$ stress.

The exposure to $\mathrm{NaCl}$ stress increased the Evans blue uptake more significantly in 'PH4CV' than in 'PH6WC', as compared with respective controls. Staining intensified with an increase in the duration of exposure to $\mathrm{NaCl}$. Control roots not treated with $\mathrm{NaCl}$ showed almost no staining. The 'PH4CV' maize seedlings turned blue after $9 \mathrm{~d}$ of $\mathrm{NaCl}$ stress, indicating that the root cells were no longer viable. The microscopic observation indicated similar results (Fig. 1, Fig. 2 Suppl.).

In the present study, the metabolic changes in two maize cultivars displayied contrasting responses to $\mathrm{NaCl}$ stress. Based on the principal component analysis, a clear separation between the NaCl-treated group and controls could be observed. Furthermore, the two cultivars were distinguished distinctly (Fig. 3 Suppl.). These results suggest that distinct metabolic programs are engaged at the different stages of $\mathrm{NaCl}$ stress and differential metabolic responses between 'PH4CV' and 'PH6WC' could be the basis of their contrasting tolerance to $\mathrm{NaCl}$ stress.

Single variable analysis was used to assess the difference in metabolites between the two groups of samples. After

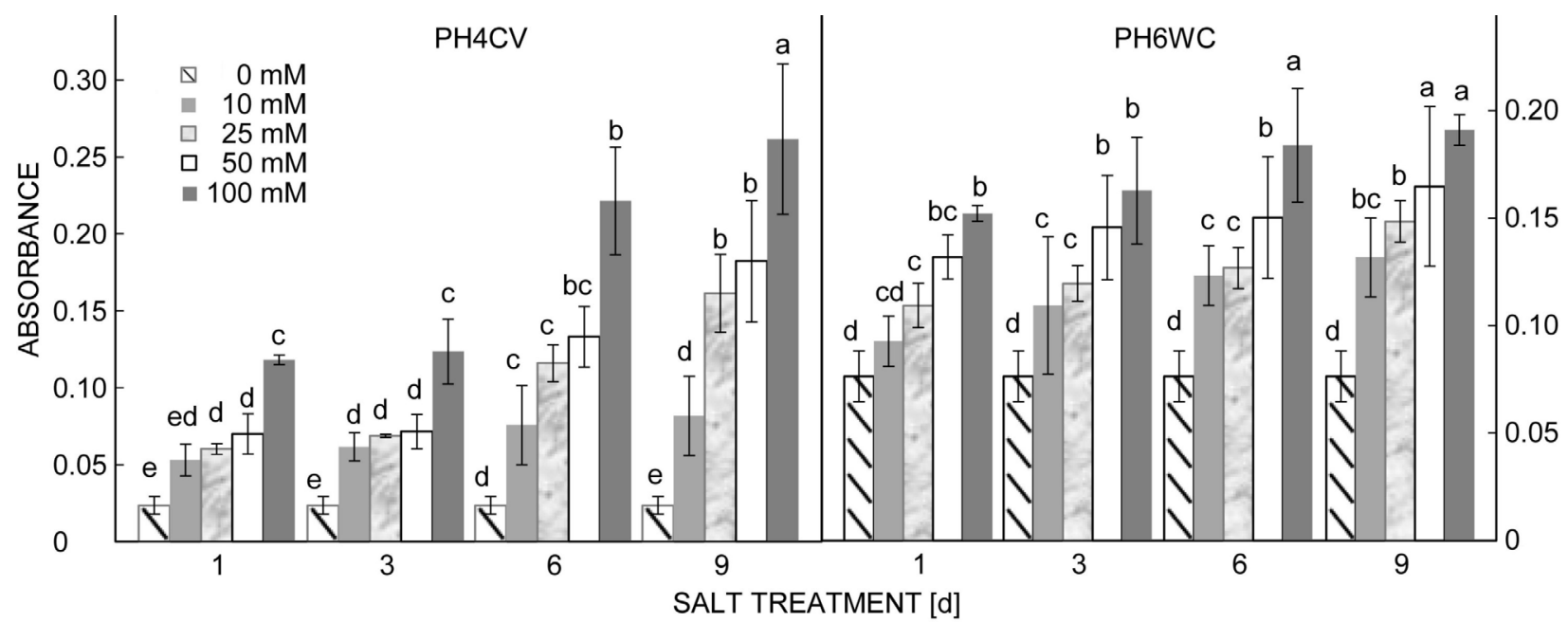

Fig. 1. The absorbance of the Evans blue solution extracted from roots of two maize cultivars under $\mathrm{NaCl}$ stress at different time points. Means \pm SEs, $n=3$, different letters indicate statistically significant differences at $P<0.05$.

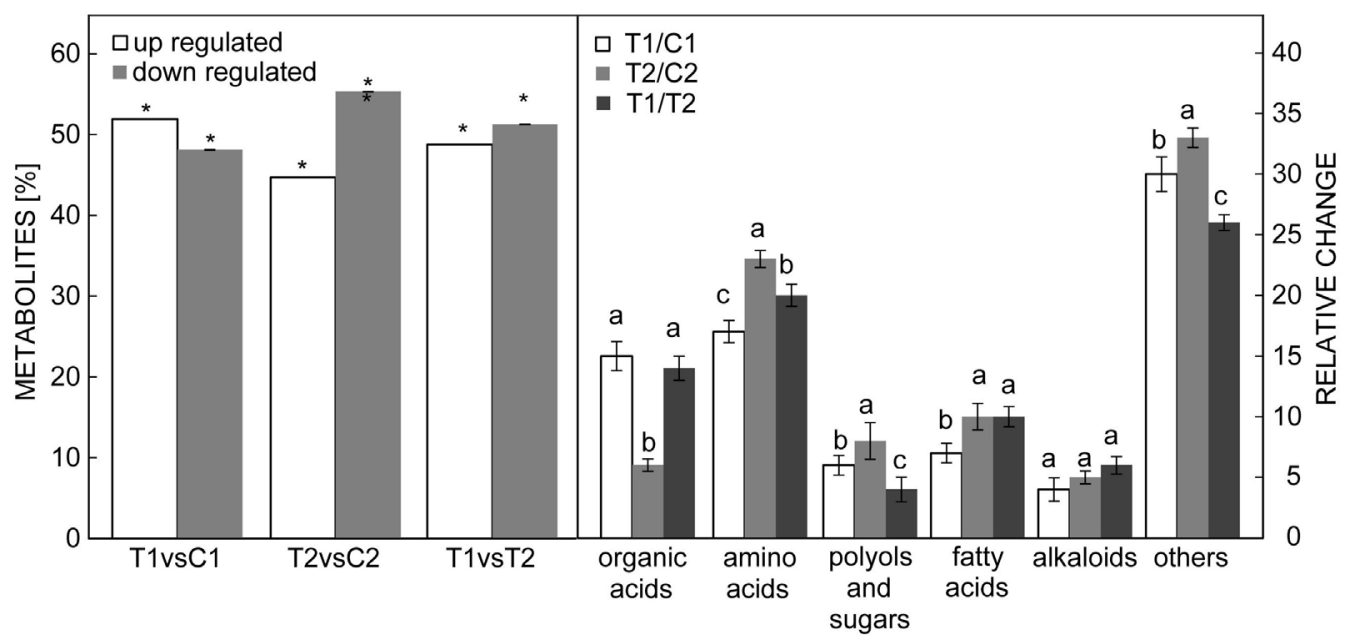

Fig. 2. Changes in the percentage of the total number of metabolites in the roots of two maize cultivars PH4CV and $\mathrm{PH} 6 \mathrm{WC}$ under $\mathrm{NaCl}$ stress. T1 - NaCl-treated 'PH4CV', C1 - 'PH4CV', T2 - NaCl-treated 'PH6WC', C2 - 'PH6WC' (right part: * indicates significance $(P<0.05),{ }^{* *}$ indicates high significance differences $(p<0.01$; left part - different letters indicate statistically significant difference at $P<0.05$. 
$6 \mathrm{~d}$ of $100 \mathrm{mM} \mathrm{NaCl}$ treatment, the metabolites in salttreated maize roots showed significant changes compared with the control group. As shown in Fig. 4 Suppl., the red dot represents the different metabolites with $F C>1.5$ and $P<0.05$. These results suggested that potential marker metabolites could be further screened.

We profiled the metabolic changes in two maize cultivars displaying contrasting responses to $\mathrm{NaCl}$ stress. Changes associated with response to $\mathrm{NaCl}$ stress were calculated as a ratio of relative metabolite concentration of the treated plants to that of the controls. The patterns varied widely, with some metabolites upregulated and some downregulated. Certain metabolites were present in mixed patterns between 'PH4CV' and 'PH6WC' following $\mathrm{NaCl}$ treatment. Dramatic variations in the significant root metabolites among the four groups, i.e., $\mathrm{C} 1, \mathrm{C} 2, \mathrm{~T} 1$, and T2 (cutoff of two-fold $\log 2, P<0.05$ ), were compared.

'PH4CV' is sensitive to $\mathrm{NaCl}$. Under $100 \mathrm{mM} \mathrm{NaCl}$ treatment; 79 compounds in its roots were identified as significant in comparisons between $\mathrm{NaCl}$-treated plants and controls with five replicates. A total of $51.9 \%$ of the metabolites were accumulated whereas $48.1 \%$ of the metabolites decreased. At least 33 metabolites showed a more than two-fold increase compared with controls. Amino acids (17), organic acids (15), polyols and sugars (6), fatty acids (7), and alkaloids (4) were the major groups of metabolites that were altered because of $\mathrm{NaCl}$ stress (Fig. 2).

'PH6WC' is tolerant of $\mathrm{NaCl}$ stress. Under $100 \mathrm{mM}$ $\mathrm{NaCl}$ treatment conditions, 85 compounds in the roots

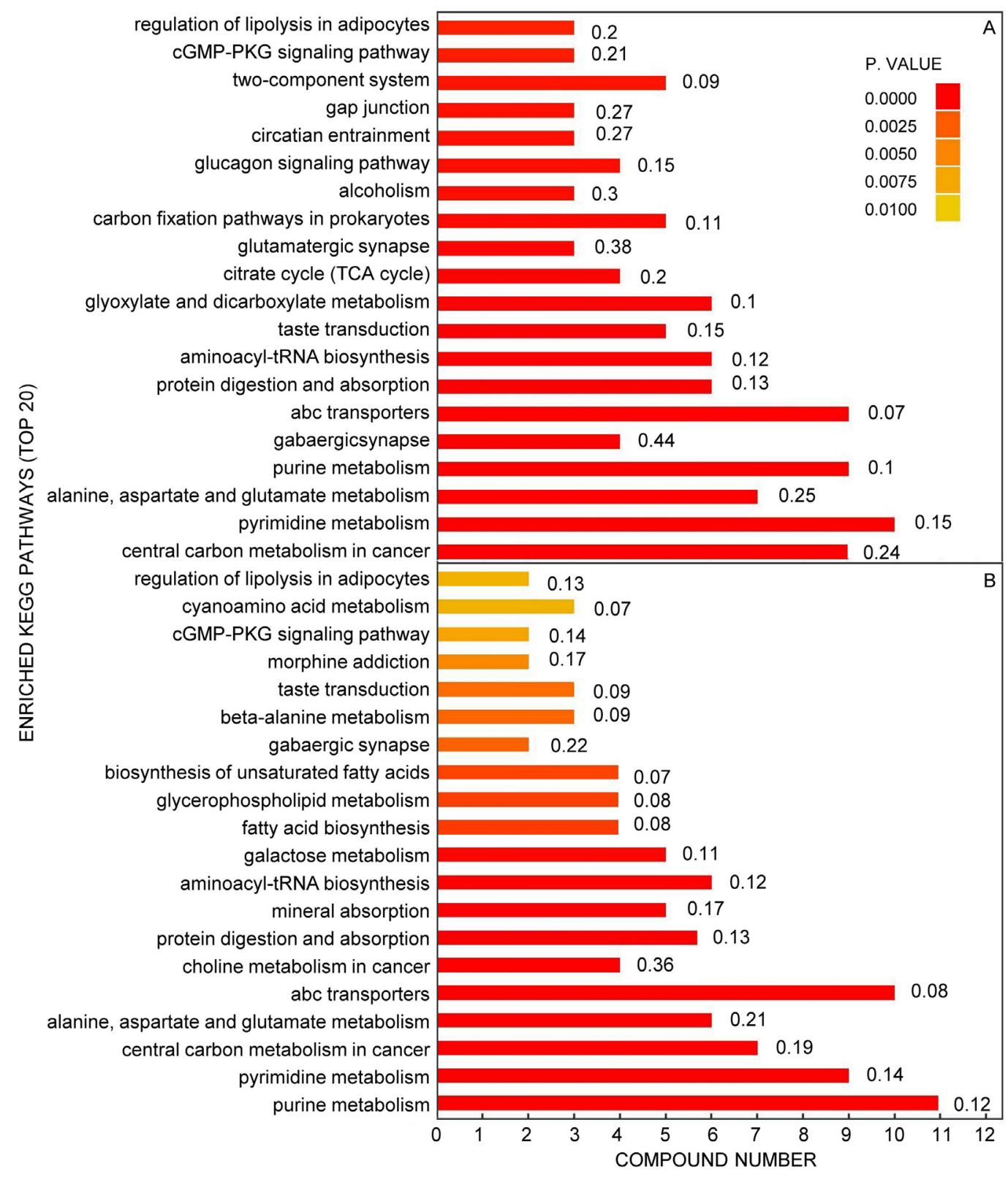

Fig. 3. The $K E G G$ pathway enrichment analysis of the differentially accumulated metabolites between maize cultivars PH4CV $(A)$ and PH6WC $(B)$ at $6 \mathrm{~d}$ under $\mathrm{NaCl}$ stress. 
were identified as significant compared with the control plants. A total of $48.75 \%$ of the metabolites were positively accumulated whereas $51.25 \%$ of the metabolites decreased. At least 47 metabolites exhibited a more than two-fold increase compared with control. Amino acids (23), delspray (10), polyols and sugars (8), organic acids (6), and alkaloids (5) are major groups of metabolites altered in 'PH4CV' and 'PH6WC' because of $\mathrm{NaCl}$ stress (Fig. 2).

The metabolite induced by $\mathrm{NaCl}$ stress in two different maize cultivars showed that 80 compounds in roots were significantly different between $\mathrm{NaCl}$ treatment and control. And $44.71 \%$ of metabolites were accumulated, 55.29 metabolites decreased. At least 23 metabolites exhibited a more than two-fold increase compared with controls. Amino acids (20), organic acids (14), delspray (10), polyols and alkaloids (6), and sugars (4) were the major groups of metabolites that were altered because of $\mathrm{NaCl}$ stress. Among these different metabolites, 30 were found in both maize varieties responding to the $\mathrm{NaCl}$ stress. These metabolites were involved in the metabolism of some basic compounds such as cis-9-palmitoleic acid, L-pyroglutamic acid, galactinol, deoxyadenosine, and adenine, suggesting that primary metabolism was relatively highly impacted by $\mathrm{NaCl}$ stress in ' $\mathrm{PH} 4 \mathrm{CV}$ ' and 'PH6WC' (Table 1 Suppl.). The trends in accumulation of the 30 metabolites were not completely consistent between 'PH4CV' and 'PH6WC'. Of the 30 metabolites, 14 were upregulated and the remaining 16 were downregulated in 'PH4CV' whereas 15 were upregulated and the remaining 15 were downregulated in 'PH6WC'. However, glucose metabolism was exclusively induced by $\mathrm{NaCl}$ in the 'PH4CV' maize seedlings whereas nucleic acid metabolism was more significant in the 'PH6WC' maize seedlings in response to $\mathrm{NaCl}$ stress, demonstrating different metabolic responses to $\mathrm{NaCl}$ stress in the different maize cultivars.

Hierarchical cluster analysis was performed to reveal the accumulation patterns of the significantly altered metabolites after the exposure of maize seedlings to $100 \mathrm{mM} \mathrm{NaCl}$ for $6 \mathrm{~d}$. All metabolite data from the roots of both cultivars under control and stress conditions were analyzed by hierarchical cluster analysis to provide a global view of the metabolite changes in 'PH4CV' and 'PH6WC' (Fig. 6 Suppl.). Clustering analysis of the metabolic profiles from the two cultivars showed that all the biological replicates were grouped together, indicating a good correlation between replicates and high reliability of our data. No clear separation between 'PH4CV' (C1) and 'PH6WC' (C2) could be observed in the control group but $\mathrm{T} 1$ and $\mathrm{T} 2$ were grouped into different clusters under $\mathrm{NaCl}$ stress. The results of ANOVA showed that, 79 metabolites were differentially accumulated between $\mathrm{C} 1$ and T1, 85 metabolites between $\mathrm{C} 2$ and $\mathrm{T} 2$, and 80 metabolites between $\mathrm{T} 1$ and $\mathrm{T} 2$. In addition, $\mathrm{NaCl}$ treatment significantly affected metabolites mainly in the classes of nucleotides and their derivatives, organic acids, amino acid derivatives, fatty acid, and alkaloids.

Correlation analysis was used to reveal relationships among metabolites. Both positive and negative correlations occurred among metabolites in both 'PH4CV' and
'PH6WC' after $\mathrm{NaCl}$ stress. Generally, high correlations observed in both 'PH4CV' and 'PH6WC' were either among amino acids or between amino acids and nucleotides or organic acids (Fig. 5 Suppl.).

\section{Discussion}

Salt stress restricts the entire growth process of plants, but seedlings are most sensitive to salt in the early stages of growth (Tounsi et al. 2017, Zezulka et al. 2019). Maize is a salt-sensitive C4 monocot crop and excessive salt concentrations in the soil lead to the abortion of maize kernels (Zhang et al. 2019b). This study probed the salt tolerance mechanism of maize by combining observations of seedling phenotype, root activity, and root metabolomics. The results indicate that treatment of maize seedlings with $\mathrm{NaCl}$ significantly inhibited their height, leaf size, stem diameter, and root growth. Furthermore, as the treatment is prolonged and $\mathrm{NaCl}$ concentration is raised, the inhibitory effects were more evident. This is consistent with the findings reported by Turk (2019).

Salt stress often induces oxidative damage, impaires membrane integrity, and causes apoptosis (Che et al. 2019, Zhang et al. 2019b). $\mathrm{NaCl}$ stress causes dehydration of the root cells as well as it damage membranes which may lead to cell death, as it was determined by Evans staining in this study. The results of Evans staining presented here concur with those of Tian (2019), who studied the effects of the herbicide quinolinic acid on maize. When confronted with this stress, root cells became less active (Sunohara and Matsumoto 2008, Yang et al. 2018, Tian et al. 2019). This confirmed that this approach was a suitable means of determining root cell viability.

The metabolomics is used to elucidate the differential metabolic reactions that result from geographical, climatic, and species-associated factors by analyzing changes in the content of small-molecule substances. Recently, many researchers have employed metabolomic approaches to study the resistance mechanisms of plants (Umair et al. 2019). The aforementioned substances are mainly organic acids, fatty acids, amino acids, polyhydric alcohols, sugars, alkaloids, and mineral elements. In present study, the content of sucrose, betanin, most amino acids, and some fatty acids tended to rise in 'PH6WC' and 'PH4CV' under $\mathrm{NaCl}$ stress. And the amount of amino and fatty acids in the roots of maize 'PH6WC' rose sharply. We may thus assume that 'PH6WC' had better osmotic adjustment under $\mathrm{NaCl}$ stress. This result is similar to the findings of Wang (2018) studying of metabolite changes in response to low-temperature stress.

The content of organic acids generally increases when plants are under stress (Yang et al. 2012). In the present study, both 1-aminocyclopropanecarboxylic acid and 16-hydroxypalmitic acid decreased in both 'PH6WC' and 'PH4CV' under $\mathrm{NaCl}$ stress, however, 'PH6WC' significantly accumulated citric acid, citraconic acid, cisaconitate, aminoadipic acid, acetyl phosphate, cinnamic acid, ect., in its roots. Organic acids not only act as the intermediates in energy cycle, but also play a role in plant 
adaptation to nutrient shortage and other abiotic stresses. As a component of the TCA cycle, citric acid can help 'PH6WC' to maintain a healthy root system and uptake nutrients and water. This is consistent with the result of Kang (2019).

The accumulation of amino acids can enhance plant stress tolerance by detoxification of ROS in photosynthetic organs, adjustment to osmotic stress, and maintenance of the intracellular pH (Yancey 1994, Hare et al. 1998, Gill and Tuteja 2010, Kang et al. 2019,). Declines in glutamic acid, purines, and pyrimidines can lead to reduced protein synthesis (Zhang et al. 2019a,b). In the present study, enhanced accumulation of amino acids in both 'PH6WC' and 'PH4CV' was noted due to the salt stress, but their relative intensity was higher in 'PH6WC' than in 'PH4CV'. L-pyroglutamic acid, cytosine, adenine, and thymine were downregulated in both maize cultivars, suggesting a negative effect of $\mathrm{NaCl}$ stress on protein synthesis in maize. Some amino acids play an adaptive role in mediating osmotic adjustment and protecting subcellular structure (He et al. 2015). Proline can remove ROS and alleviate their effects on cell proteins and membranes (Tang et al. 2019). Comparing the two maize genotypes revealed higher tendencies for the upregulation of proline in the roots of 'PH6WC', which testifies that $\mathrm{NaCl}$ stress can induce salt-tolerant maize to accumulate more proline to reduce membrane damage. This finding is similar to that of Saeedipour (2013), who indicated that higher proline content was found in maize seedlings under a low water potential. This result also supports the evidence that proline alleviates the deleterious effect of stress (Saeedipour 2013). The content of $\gamma$-aminobutyric acid (GABA) also increased under $\mathrm{NaCl}$ stress. GABAregulated developmental processes, $\mathrm{pH}$, and stress tolerance (Umair et al. 2019).

Fatty acids are constituents of plant membranes. Under $\mathrm{NaCl}$ stress, the content of fatty acids decrease because of membrane damage (Yang et al. 2012, Hu et al. 2015). In the present study, when compared with the control group, the content of fatty acids in the roots decreased in 'PH4CV' and increased in 'PH6WC' under $\mathrm{NaCl}$ treatment. This may suggest that 'PH6WC' can respond quickly to $\mathrm{NaCl}$ stress by accumulating oleic acid, citrate, myristic acid, 21-hydroxypregnenolone, and GABA, ect., to alleviate the damage of membranes caused by $\mathrm{NaCl}$ exposure.

Saccharides participate in the metabolism of galactose. They can also promote water absorption in seedling roots, regulate the osmotic potential of plant cells, and their content reflect the regulation of photosynthesis. Saccharide content indicates that photosynthesis has been affected under certain conditions (Umair et al. 2019). In this study, both maize species upregulated content of sucrose and betanin in response to $\mathrm{NaCl}$ treatment, indicating that the roots of maize seedling can adjust the water potential through saccharide accumulation. Hu (2019) studied the effect of stress on saccharide-related genes and discovered that saccharides could respond to salt stress. The comparison of maize cultivars indicated that salt-sensitive 'PH4CV' accumulated more saccharides whereas salt-resistant
'PH6WC' gathered more amino acids, organic acids, and fatty acids for osmotic adjustment. It can be assumed, that in the roots of salt-sensitive ' $\mathrm{PH} 4 \mathrm{CV}$ ' galactose metabolism is inhibited and photosynthesis is reduced. Compared with the control groups, the NaCl-treated groups of both cultivars exhibited lower concentrations of $\mathrm{Mg}^{2+}$ but 'PH6WC' exhibited less decline in $\mathrm{Mg}^{2+}$. This suggests that 'PH6WC' maize can more effectively absorb $\mathrm{Mg}^{2+}$ and decelerate chlorophyll degradation. This is consistent with the findings of Jin (2017), who revealed that $\mathrm{NaCl}$ stress can affect absorption of $\mathrm{Mg}^{2+}$ and thus their photosynthesis. He also verified that low $\mathrm{Mg}^{2+}$ stress can affect chlorophyll content and organic acid accumulation in potato plantlets.

On the basis of the analysis of differential metabolites, this paper studied differential metabolic pathways and determined that maize adapts to the $\mathrm{NaCl}$ stress by initiating the metabolism of purines, pyrimidines, alanine, aspartic acid, and glutamic acid, synthesis of aminoacyl t-RNA, protein digestion and absorption, and adipocyte esterlysis and subsequently regulating the synthesis and decomposition of purines, pyrimidines, amino acids, and fatty acids (Fig. 3). Wang (2019) investigated the temporal metabolic responses to salinity in hulls of barley. They performed a widely targeted metabolomic analysis of 72 leaf samples from two contrasting cultivars and identified 642 compounds, $57 \%$ of which were affected by salt stress in the two cultivars, these were principally amino acids and their derivatives, organic acids, nucleotides, and flavonoids. This study reveals that under $\mathrm{NaCl}$ stress, salt-sensitive and salt-tolerant maize cultivars are not metabolically consistent with each other. As 'PH4CV' underwent a significant change in the citrate cycle and metabolism of glyoxylic acid and dicarboxylic acid, 'PH6WC' changed mainly galactose metabolism, mineral absorption, biosynthesis and metabolism of fatty acids, and glyceryl phosphatide metabolism. By studying the response maize to Cd stress, Lian (2019) found that galactose metabolism, the citrate cycle, and the metabolism of related amino acids could relieve $\mathrm{Cd}$ poisoning of maize, which is similar to the findings of this paper. Changes in the content of saccharides, fatty acids, unsaturated fatty acids, and $\mathrm{Mg}^{2+}$ are all closely related to the salt tolerance of maize.

\section{Conclusions}

The results of the experiments conducted in this study revealed that the salt-tolerant and salt-intolerant maize cultivars displayed significant differences in response to treatment with $100 \mathrm{mM} \mathrm{NaCl}$ for $6 \mathrm{~d}$. When compared with the control groups, content of organic acids, $\mathrm{Mg}^{2+}$, L-pyroglutamic acid, purines, and pyrimidines decreased while content of sucrose, betanin, most amino acids, and some fatty acids increased in treated groups. The salt stress reduced the chlorophyll content and photosynthetic rate. However, maize seedlings accumulate osmotic adjustment substances to lower the water potential and to eliminate 
complete dehydration. The salt sensitive cultivar PH4CV was not able to regulate metabolic pathways as effectively as salt-resistant cultivar PH6WC.

\section{References}

Abd El Gawad, H., Zinta, G., Hegab, M.M., Pandey, R., Asard, H., Abu El Soud, W.: High salinity induces different oxidative stress and antioxidant responses in maize seedlings organs. Front. Plant Sci. 7: 276, 2016.

Baker, C.J., Mock, N.M.: An improved method for monitoring cell death in cell suspension and leaf disc assays using evans blue. - Plant Cell Tissue Organ Cult. 39: 7-12, 1994.

Cai, R., Dai, W., Zhang, C., Wang, Y., Wu, M., Zhao, Y., Ma, Q., Xiang, Y., Cheng, B.: The maize WRKY transcription factor $Z m W R K Y 17$ negatively regulates salt stress tolerance in transgenic Arabidopsis plants. - Planta 246: 1215-1231, 2017.

Carillo, P., Cirillo C., De Micco V., Arena C., De Pascale S., Rouphael Y.: Morpho-anatomical, physiological and biochemical adaptive responses to saline water of Bougainvillea spectabilis Willd. trained to different canopy shapes. - Agr. Water Manage. 212: 12-22, 2019.

Carillo, P., Woodrow, P., Raimondi, G., El-Nakhel, C., Pannico, A., Kyriacou, M.C., Colla, G., Mori M., Giordano, M., De Pascale, S., Rouphael, Y.: Omeprazole promotes chloride exclusion and induces salt tolerance in greenhouse basil. Agronomy 9: 355, 2019.

Ceccarini, C., Antognoni, F., Biondi, S., Fraternale, A.: Polyphenol-enriched spelt husk extracts improve growth and stress-related biochemical parameters under moderate salt stress in maize plants. - Plant Physiol. Biochem. 141: 95-104, 2019.

Chalivendra, S.C., De Robertis, C., Chang, P.K, Damann, K.E.: Cyclopiazonic acid is a pathogenicity factor for Aspergillus flavus and a promising target for screening germplasm for ear rot resistance. - Mol. Plant-Microbe Interact. 30: 361-373, 2017.

Che, B., Cheng, C., Fang, J., Liu, Y., Jiang, L., Yu, B.: The recretohalophyte tamarix TrSOS1 gene confers enhanced salt tolerance to transgenic hairy root composite cotton seedlings exhibiting virus-induced gene silencing of GhSOS1. - Int. J. mol. Sci. 20: 2930, 2019.

Du, X., Wang, G., Ji, J., Shi, L., Guan, C., Jin, C.: Comparative transcriptome analysis of transcription factors in different maize varieties under salt stress conditions. - Plant Growth Regul. 81: 183-195, 2017.

El-Katony, T.M., El-Bastawisy, Z.M., El-Ghareeb, S.S.: Timing of salicylic acid application affects the response of maize (Zea mays L.) hybrids to salinity stress. - Heliyon 5: 01547, 2019.

Farooq, M., Hussain, M., Wakeel, A., Siddique, K.H.M.: Salt stress in maize effects resistance mechanisms and management: a review. - Agron. Sustain. 35: 461-481, 2015.

Flowers, T.J., Munns, R., Colmer, T.D.: Sodium chloride toxicity and the cellular basis of salt tolerance in halophytes. - Ann. Bot. 115: 419-431, 2015.

Gao, Y., Lu, Y., Wu, M., Liang, E., Li, Y., Zhang, D., Yin, Z., Ren, X., Dai, Y., Deng, D., Chen, J.: Ability to remove $\mathrm{Na}^{+}$and retain $\mathrm{K}^{+}$correlates with salt tolerance in two maize inbred lines seedlings. - Front. Plant Sci. 7: 1716, 2016.

Gill, S.S., Tuteja, N.: Reactive oxygen species and antioxidant machinery in abiotic stress tolerance in crop plants. - Plant Physiol. Biochem. 48: 909-930, 2010.

Gholami, M., Bahabadi, S.E., Ghanati, F., Borojeni, L.Y.: Stereospecific transcript regulation of the polyamine biosynthesis genes by enantiomers of ornithine in tobacco cell culture. Iran. J. Biotechnol. 16: 148-153, 2018.

Hare, P.D., Cress, W.A., Van Staden, J.: Dissecting the roles of osmolyte accumulation during stress. - Plant Cell Environ. 21: $535-553,1998$.

He, X., Chen, Z., Wang, J., Li, W., Zhao, J., Wu, J., Wang, Z., Chen, X.: A sucrose:fructan-6-fructosyltransferase (6-SFT) gene from Psathyrostachys huashanica confers abiotic stress tolerance in tobacco. - Gene 570: 239-247, 2015.

Hu, B., Wu, H., Huang, W., Song, J., Zhou, Y., Lin, Y.: Sweet gene family in Medicago truncatula: genome-wide identification, expression and substrate specificity analysis. - Plants 8: 338, 2019.

Hu, L.X., Zhang, P.P., Jiang, Y., Fu, J.M.: Metabolomic analysis revealed differential adaptation to salinity and alkalinity stress in Kentucky bluegrass (Poa pratensis). - Plant mol. Biol. Rep. 33: 56-68, 2015.

Issaoui, K.H., Ferchichi, K., Saif, S., Abdelly, T., Siddique, C., Kadambot, H.M., Cruz, C.: Interactive effects of salinity and nitrogen forms on plant growth, photosynthesis and osmotic adjustment in maize. - Plant Physiol. Biochem. 139: 171-178, 2019.

Jiang, J.L., Tian, Y., Li, L., Yu, M., Hou, R.P., Ren, X.M.: $\mathrm{H}_{2} \mathrm{~S}$ alleviates salinity stress in cucumber by maintaining the $\mathrm{Na}^{+}$/ $\mathrm{K}^{+}$balance and regulating $\mathrm{H}_{2} \mathrm{~S}$ metabolism and oxidative stress response. - Front. Plant Sci. 10: 678, 2019.

Jin, J., Cui, H., Lv, X., Yang, Y., Wang, Y., Lu, X.: Exogenous $\mathrm{CaCl}_{2}$ reduces salt stress in sour jujube by reducing $\mathrm{Na}^{+}$and increasing $\mathrm{K}^{+}, \mathrm{Ca}^{2+}$, and $\mathrm{Mg}^{2+}$ in different plant organs. - $\mathrm{J}$. hort. Sci. Biotechnol. 92: 98-106, 2017.

Juhos, K., Szabó, S., Ladányi, M.: Influence of soil properties on crop yield: a multivariate statistical approach. - Int. Agrophysics 29: 433-440, 2015.

Jung, S., Hütsch, B.W., Schubert, S.: Salt stress reduces kernel number of maize by inhibiting plasma membrane $\mathrm{H}^{+}$-ATPase activity. - Plant Physiol. Biochem. 113: 198-207, 2017.

Lian, J., Zhao, L., Wu, J., Xiong, H., Bao, Y., Zeb, A., 'Tang, J., Liu, W.: Foliar spray of $\mathrm{TiO}_{2}$ nanoparticles prevails over root application in reducing $\mathrm{Cd}$ accumulation and mitigating $\mathrm{Cd}$ induced phytotoxicity in maize (Zea mays L.). - Chemosphere 239: 124794, 2019.

Kang, Z., Babar, M.A., Khan, N., Guo, J., Khan, J., Islam, S., Shrestha, S., Shahi, D.: Comparative metabolomic profiling in the roots and leaves in contrasting genotypes reveals complex mechanisms involved in post-anthesis drought tolerance in wheat. - PloS ONE 14: e0213502, 2019.

Luo, M., Zhao, Y., Wang, Y., Shi, Z., Zhang, P., Zhang Y., Song, W., Zhao, J.: Comparative proteomics of contrasting maize genotypes provides insights into salt-stress tolerance mechanisms. - J. Proteome Res. 17: 141-153, 2017 a.

Luo, M., Zhao, Y., Zhang, R., Xing, J., Duan, M., Li, J., Wang, N., Wang, W., Zhang, S., Chen, Z., Zhang, H., Shi, Z., Song, W., Zhao, J.: Mapping of a major QTL for salt tolerance of mature field-grown maize plants based on SNP markers. BMC Plant Biology 17: 140, $2017 \mathrm{~b}$.

Richter, J.A., Erban, A., Kopka, J., Zörb, C.: Metabolic contribution to salt stress in two maize hybrids with contrasting resistance. - Plant Sci. 233: 107-115, 2015.

Rouf Shah, T., Prasad, K., Kumar, P.: Maize - a potential source of human nutrition and health: a review. - Cogent Food Agr. 2: 1166995, 2016.

Saeedipour, S.: Relationship of grain yield, ABA and proline accumulation in tolerant and sensitive wheat cultivars as affected by water stress. - Proc. nat. Acad. Sci. India 83: 311315,2013

Shabala, L., Zhang, J., Pottosin, I., Bose, J., Zhu, M., Fuglsang, 
A.T., Velarde-Buendia, A., Massart, A., Hill, C.B., Roessner, U., Bacic, A., Wu, H., Azzarello, E., Pandolfi, C., Zhou, M., Poschenrieder, C., Mancuso, S., Shabala, S.: Cell-type-specific $\mathrm{H}^{+}$- ATPase activity in root tissues enables $\mathrm{K}^{+}$retention and mediates acclimation of barley (Hordeum vulgare) to salinity stress. - Plant Physiol. 172: 2445-2458, 2016.

Shelke, D.B, Pandey, M., Nikalje, G.C., Zaware, B.N., Suprasanna, P., Nikam, T.D.: Salt responsive physiological, photosynthetic and biochemical attributes at early seedling stage for screening soybean genotypes. - Plant Physiol. Biochem. 118: 519-528, 2017.

Singh, S., Parihar, P., Singh, R., Singh, V.P., Prasad, S.M.: Heavy metal tolerance in plants: role of transcriptomics, proteomics, metabolomics, and ionomics. - Front Plant Sci. 6: 1143, 2015.

Song, Y., Yang, X., Yang, S., Wang, J.: Transcriptome sequencing and functional analysis of Sedum lineare Thunb. upon salt stress. - Mol. Genet. Genomics 294: 1441-1453, 2019.

Sunohara, Y., Matsumoto, H.: Quinclorac-induced cell death is accompanied by generation of reactive oxygen species in maize root tissue. - Phytochemistry 69: 2312-2319, 2008.

Tang, H., Niu, L., Wei, J., Chen, X.: Phosphorus limitation improved salt tolerance in maize through tissue mass density increase, osmolytes accumulation, and $\mathrm{Na}^{+}$uptake inhibition. - Front. Plant Sci. 10: 856, 2019.

Tian, X., Li, S., Zeng, Q., Huang, W.: Relationship between loss of desiccation tolerance and programmed cell death (PCD) in mung bean (Vigna radiata) seeds. - PloS ONE, 14: e0218513, 2019.

Tounsi, S., Feki, K., Hmidi, D., Masmoudi, K., Brini, F.: Salt stress reveals differential physiological, biochemical and molecular responses in T. monococcum and T. durum wheat genotypes. - Physiol. mol. Biol. Plants 23: 517-528, 2017.

Trevisan, S., Trentin, A.R., Ghisi, R., Masi, A., Quaggiotti, S.: Nitrate affects transcriptional regulation of UPBEAT1 and ROS localisation in roots of Zea mays L. - Physiol. Plant. 166: 794-811, 2019.

Turk, H.: Chitosan-induced enhanced expression and activation of alternative oxidase confer tolerance to salt stress in maize seedlings. - Plant Physiol. Biochem. 141: 415-422, 2019.

Umair, M., Sun, N., Du, H., Yuan, J., Abbasi, A.M., Wen, J., Yu, W., Zhou, J., Liu, C.: Differential metabolic responses of shrubs and grasses to water additions in arid karst region, southwestern China. - Sci. Rep. 9: 9613, 2019.

Wang, C., Lu, G., Hao, Y., Guo, H., Guo, Y., Zhao, J., Cheng, H.: ABP9, a maize bZIP transcription factor, enhances tolerance to salt and drought in transgenic cotton. - Planta 246: 453-469, 2017.

Wang, T.: Proteomics and Metabolomics Analysis to Cold Stress in Two Desert Chenopodiaceae Plant Seedlings. - Shihezi University, Shizei 2018.

Wang, Y., Zeng, X., Xu, Q., Mei, X., Yuan, H., Jiabu, D., Sang, Z., Nyima, T.: Metabolite profiling in two contrasting Tibetan hulless barley cultivars revealed the core salt-responsive metabolome and key salt-tolerance biomarkers. - AoB Plants 11: plz021, 2019.

Yancey, P.H.: Compatible and counteracting solutes. - In: Strange, K. (ed.): Cellular and Molecular Physiology of Cell Volume Regulation. Pp. 81-109. CRC Press, Boca Raton 1994.

Yang, G., Gao, X., Ma, K., Li, D., Jia, C., Zhai, M., Xu, Z.: The walnut transcription factor JrGRAS2 contributes to high temperature stress tolerance involving in Dof transcriptional regulation and HSP protein expression. - BMC Plant Biol. 18: 367, 2018.

Yang, S., Zhang, H., Liu, T.: Effect of salt stress on osmotic adjustment substances in plants. - Forest Res. 25: 269-277, 2012.

You, J., Zhang, Y., Liu, A., Li, D., Wang, X., Dossa, K., Zhou, R., Yu, J., Zhang, Y., Wang, L., Zhang, X.: Transcriptomic and metabolomic profiling of drought-tolerant and susceptible sesame genotypes in response to drought stress. - BMC Plant Biol. 19: 267, 2019.

Zezulka, Š., Kummerová, M., Babula, P., Hájková, M., Oravec, M.: Sensitivity of physiological and biochemical endpoints in early ontogenetic stages of crops under diclofenac and paracetamol treatments.- Environ. Sci. Pollut. Res. Int. 26: 3965-3979, 2019.

Zhang, H., Ji, S., Guo, R., Zhou, C., Wang, Y., Fan, H., Liu, Z.: Hydrophobin HFBII-4 from Trichoderma asperellum induces antifungal resistance in poplar. - Braz. J. Microbiol. 50: 603612, 2019a.

Zhang, H., Lu, L., Zhao, X., Zhao, S., Gu, X., Du, W., Wei, H., Ji, R., Zha, L.: Metabolomics reveals the "invisible" responses of spinach plants exposed to $\mathrm{CeO} 2$ nanoparticles. - Environ. Sci. Technol. 53: 6007-6017, 2019b.

Zhang, M., Cao, Y., Wang, Z., Wang, Z.Q., Shi, J., Liang, X., Song, W., Chen, Q., Lai, J., Jiang, C.: A retrotransposon in an HKT1 family sodium transporter causes variation of leaf $\mathrm{Na}^{+}$exclusion and salt tolerance in maize. - New Phytol. 217: 1161-1176, 2018 